\title{
研究通讯
}

\section{双平稳随机时变信道最大似然序列 检测的概率收敛定理}

本文首先利用平稳 $\mathrm{K}$ 相依随机变量序列的中心 极限定理, 给出了双平稳随机时变信道最大似然序 列检测定理一个新的、准确的叙述和证明. 然后, 导 出了序列检测概率收敛速度的定量计算公式. 最 后,对序列检测技术的某些重要问题进行了讨论.所 得的主要结果可以写成

定王 1 设 $I_{0} 、 I_{1} 、 \cdots 、 I_{K+k}$ 和 $r_{0} 、 r_{1}^{\prime}, \cdots, I_{K+k}^{\prime}$ 是二个任意的信息序列, $\tilde{s}_{0} 、 \tilde{s}_{1} 、 \cdots 、 \tilde{s}_{\boldsymbol{K}+\boldsymbol{k}}$ 是接收信 号的样值序列 $(k=-1,0,1,2, \cdots)$. 如果我们定 义

$$
L_{K+k}\left(I_{0}, I_{1}, \cdots, I_{K+k}\right)=\sum_{n=0}^{K+k} \ln P\left(\tilde{S}_{n} / I_{n-K+1}, \cdots, I_{n}\right)
$$

和

$$
Z_{n}=\ln \frac{P\left(\tilde{S}_{n} / I_{n-K+1}, \cdots, I_{n}^{\prime}\right)}{P\left(\tilde{S}_{n} / I_{n-K+1}, \cdots, I_{n}\right)}
$$

那么

$$
\lim _{k \rightarrow \infty} P\left(\sum_{n=0}^{K+k} z_{n} \leqslant 0\right)=0.5
$$

或

$$
\lim _{k \rightarrow \infty} P\left(\sum_{n=0}^{K+k} Z_{n}>0\right)=0.5,
$$

其中, 对每个 $m=0,1,2, \cdots, I_{m}$ 是以等概率 $\frac{1}{2}$ 取 值 +1 和 -1 的随机变量, $\tilde{s}_{m} 、 \tilde{s}_{m+1} 、 \cdots 、 \tilde{s}_{m+K_{-1}}$ 都 与 $I_{m}$ 有关. $K$ 是与信道有关的一个正整数, 而 $L_{K+k}$ $\left(I_{0}, I_{1}, \cdots, I_{K+k}\right)$ 是似然函数.

定珽 2 在基带序列检测技术中，对任意正 整数 $m$ 和 $k=-1,0,1,2, \cdots$, 可以判决每个信息序 列 $I_{0} 、\left(I_{0}, I_{1}\right) 、\left(I_{0}, I_{1}, I_{2}\right) 、 \cdots$ 的概刺全是 $2^{(1-2} \mathrm{K}-1$, 另一方面，所有信息序列 $I_{0} 、\left(I_{0}, I_{1}\right) 、 \cdots 、\left(I_{0} 、 I_{1}, \cdots\right.$, $\left.l_{m-2}\right)$ 都不能判决的概率 $p_{m, K}$ 为

$$
p_{m, K}=\left[1-2^{\left(1-2^{K-1}\right)}\right]^{m} .
$$

戴耀森

(杭州大学数学系)

\section{一些 集 合 的 基 数 (III)}

在考存数学中某些基本概念时, 我们又得到了 几个初步的结果:

设 $X$ 是固定的集合且为无穷, $F$ 是域且为适当 取定的、有无穷多个元亲的域,在这样的条件下我们 进一步假定，并命:

1. $F_{0}=\{(x, f, g, h): X$ 在 $f, g, h$ 下成为有 序域\}

2. $F_{c}=\{(X, f, g, h): X$ 在 $f, g, h$ 下成为序 完全的有序域 $\}$, 并且假定 $|X| \geqslant|R|=$ 连续统的 基数.

3. $V=\{(x, f, F . k): x$ 是 $F$ 上的在 $f, k$ 下成 为向量空间 $\}$,并且假定 $|x| \geqslant|F|$.

4. $A=\{(X, f, g ; F ; k): X$ 是 $F$ 上的在 $f, g, k$
之下成为代数 $\}$, 还要假定 $|X| \geqslant|F|$.

5. $V_{i}=\{(X, f ; F ; k ; p): X$ 是 $F$ 上的在 $f, k$, $p$ 下成为一般的内积空间 $\}$, 并且假定 $|X|=|F|$.

6. $\boldsymbol{H}=\{(X, f ; F ; k ; p): X$ 是 $F$ 上的在 $f, k, p$ 下成为一般的 Hilbert 空间\},并且假定 $|X|=|F| \geqslant$ $|R|=$ 连续统的基数.

对于以上的六个集合, 其基数均为 $2^{|x|}$, 即它们 均能取得最大值、取得其上界值 $2^{|x|}$. 在证明的过 程中我们使用了选择公理。

杨安洲

(北京工业大学数学系) 